\title{
Constraints of beyond Standard Model parameters from the study of neutrinoless double beta decay
}

\author{
Sabin Stoica ${ }^{1,2, a}$ \\ ${ }^{1}$ Horia Hulubei Foundation, P.O. Box MG12, and \\ ${ }^{2}$ Horia Hulubei Institute of Physics and Nuclear Engineering, P.O. Box MG6, \\ 077125 Bucharest-Magurele, Romania
}

\begin{abstract}
Neutrinoless double beta $(0 v \beta \beta)$ decay is a beyond Standard Model (BSM) process whose discovery would clarify if the lepton number is conserved, decide on the neutrinos character (are they Dirac or Majorana particles?) and give a hint on the scale of their absolute masses. Also, from the study of $0 v \beta \beta$ one can constrain other BSM parameters related to different scenarios by which this process can occur. In this paper I make first a short review on the actual challenges to calculate precisely the phase space factors and nuclear matrix elements entering the $0 v \beta \beta$ decay lifetimes, and I report results of our group for these quantities. Then, taking advance of the most recent experimental limits for $0 v \beta \beta$ lifetimes, I present new constraints of the neutrino mass parameters associated with different mechanisms of occurrence of the $0 v \beta \beta$ decay mode.
\end{abstract}

\section{Introduction}

Despite the fact that neutrinos are among the most abundant particles in the universe, their fundamental properties as the absolute mass and mass hierarchy, their character (are they Dirac or Majorana particles?), the number of neutrino flavors other than the three active ones, etc. are still unknown. There are different processes/measurements from which one can get information about these issues, one of them being the neutrinoless double beta $(0 v \beta \beta)$ decay [1] -[6]. Double beta decay (DBD) is the rarest spontaneous nuclear decay measured until now, by which an e-e nucleus transforms into another e-e nucleus with its nuclear charge changed by two units. It occurs whatever single beta decays can not occur due to energetic reasons or it is highly forbidden by angular momentum selection rules. Usually, the DBD process occurs with the emission of two neutrinos or two anti-neutrinos besides two positrons or two electrons, and hence with the lepton number conservation (LNC). However, since the LNC is not a consequence of a known gauge symmetry, BSM theories allow DBD to occur without emission of anti-neutrinos/neutrinos (the so called $0 v \beta \beta$ decay modes), as well. In this contribution I refer to the $0 v \beta \beta$ with the emission of two electrons in the final states. This process can provide us with decisive information about the neutrino character, absolute mass of neutrino and mass hierarchy, and it still is at present the most sensitive process to check the lepton number conservation. The lifetime expression of this process can be factorized in a good approximation as a product of three factors: the phase space factor (PSF) that embeds the distortion of the electron wave functions (w.f.) by the nuclear

ae-mail: stoica@nipne.ro 
Coulomb potential, the nuclear matrix elements (NME) that depend on the nuclear structure of the nuclei involved in decay and a BSM factor related to the mechanism that contributes to $0 v \beta \beta$. At present, the NME are currently computed with several nuclear structure methods, such as the proton-neutron Quasi Random Phase Approximation (pnQRPA) [7]-[10], Shell Model(ShM) [11]-[12], Interacting Boson Approximation (IBA) [13]-[14], Projected Hartree Fock Bogoliubov (PHFB) [15] and Energy Density Functional (EDS) method [16]. The computation of the NME brings the largest uncertainties in the theoretical study of double beta decay (DBD) process. Each method has its advantages and disadvantages and there has been and still is a long debate in literature on their calculations. Unlike the NME, the PSF have been considered to be computed with enough precision [17]-[19]. However, recently, they were recalculated within an improved approach by using exact electron w.f., solution of the Dirac equations, and taking into account finite nuclear size and electron screening effects [20]. The authors found differences between their results and those calculated previously with approximate electron w.f., especially for heavier nuclei. We also recalculated the PSF by developing new accurate routines for computing the relativistic (Dirac) electron w.f. and for integrating the PSF expressions. In addition to the previous similar calculations, we use a Coulomb potential derived from a realistic proton density distribution in the daughter nuclei [21]-[22]. Hence, the NME and PSF are theoretical key quantities for predicting $0 v \beta \beta$ decay half-lives. On the other hand, because the $0 v \beta \beta$ decay has not been discovered so far, there are only experimental lifetime limits for this process. Using these limits and precise calculations of the PSF and NME, one can derive upper limits of the BSM parameters associated with specific decay mechanisms.

\section{Theoretical formalism}

We shortly present the theoretical framework for the derivation of neutrino mass parameters from the $0 v \beta \beta$ decay analysis. In the hypothesis that this DBD mode occurs through two mechanisms, namely i) light left- handed $(\mathrm{LH})$ neutrinos and ii) heavy right-handed $(\mathrm{RH})$ neutrinos, between two nucleons inside the nucleus, the contribution of the two mechanisms do not interfere with one another and the lifetime formula can be expressed as a sum of two terms:

$$
\left(T_{1 / 2}^{0 v}\right)^{-1}=G^{0 v}\left(Q_{\beta \beta}, Z\right)\left(\left|M_{v}^{0 v}\right|^{2}\left(\eta_{v}\right)^{2}+\left|M_{N}^{0 v}\right|^{2}\left(\eta_{N}\right)^{2}\right)
$$

where $G^{0 v}$ is the PSF for this decay mode and depends on the energy decay $Q_{\beta \beta}$ and nuclear charge $Z$. $M_{v, N}^{0 v}$ are the NME for the light LH $(v)$ and heavy $\mathrm{RH}(N)$ neutrino exchange mechanisms, respectively, and $\langle\eta\rangle$ are the neutrino mass parameters which are related to the neutrino masses:

$$
<\eta_{v}>=\left(\frac{\left\langle m_{v}\right\rangle}{m_{e}}\right)^{2}
$$

with

$$
<m_{v}>=\sum_{i}^{\text {light }}\left|U_{e i}\right|^{2} m_{i} e^{i \phi_{i}}(3)
$$

for the "light" mechanism and

$$
<\eta_{N}>=\sum_{k}^{\text {heavy }}\left|U_{e k}\right|^{2} \frac{m_{p}}{m_{N}} e^{i \phi_{k}}(4)
$$

for the "heavy" mechanism. In the above formulas the $m_{i}$ and $m_{N}$ are the neutrino (light, heavy) masses, $\phi(i, k)$ Majorana phases, $m_{p}$ the proton mass and the $U_{e(i, k)}$ are the mixing matrix elements 
associated to LH weak interactions. We adopted the hypothesis that only one flavor of "'heavy"' neutrino exist.

As seen, for deriving the $\langle\eta\rangle$ parameters we need accurate calculations of both PSF and NME, for each mechanism and each isotope for which the experimental lifetime limits exist.

For the $0 v \beta \beta$ decay, the expression of the PSF is [20]-[21]:

$$
G_{0 v}=\frac{2}{4 g_{A}^{4} R^{2} \ln 2} \int_{m_{e} c^{2}}^{T_{0}-m_{e} c^{2}} f_{11}^{(0)} w_{0 v} d \epsilon_{1}
$$

where

$$
w_{0 v}=\frac{g_{A}^{4}\left(G \cos \theta_{C}\right)^{4}}{16 \pi^{5}}\left(m_{e} c^{2}\right)^{2}\left(\hbar c^{2}\right)\left(p_{1} c\right)\left(p_{2} c\right) \epsilon_{1} \epsilon_{2}
$$

In the above formulas $g_{A}$ is the axial-vector coupling constant, $R$ is the nuclear radius, $G=1.16637 \times$ $10^{-5} \mathrm{GeV}^{-2}$ is the Fermi constant, $\theta_{C}$ is the Cabbibo angle, $m_{e}$ is the electron rest mass, while $p_{1}$, $p_{2}$ and $\epsilon_{1}, \epsilon_{2}$ are the electron momenta and electron energies, respectively. It is worth to note that in Eq.(5) the PSF expression was redefined, such that this quantity be independent on the $g_{A}$ constant and correlates (by dividing by $4 R^{2}$ ) the dimension of $G_{0 v}$ with the NME which are dimensionless, such that the PSF be expressed in $\left[\mathrm{yr}^{-1}\right]$.

The electron phase factors $f_{j k}^{(0)}$ have the expressions:

$$
f_{11}^{(0)}=\left|f^{-1-1}\right|^{2}+\left|f_{11}\right|^{2}+\left|f_{1}^{-1}\right|^{2}+\left|f_{1}^{-1}\right|^{2}
$$

with

$$
f^{-1-1}=g_{-1}\left(\epsilon_{1}\right) g_{-1}\left(\epsilon_{2}\right) ; f_{11}=f_{1}\left(\epsilon_{1}\right) f_{1}\left(\epsilon_{2}\right), f_{1}^{-1}=g_{-1}\left(\epsilon_{1}\right) f_{1}\left(\epsilon_{2}\right) ; f_{1}^{-1}=f_{1}\left(\epsilon_{1}\right) g_{1}\left(\epsilon_{2}\right)
$$

The radial functions $f$ and $g$ are solutions of the Dirac equations [20]), [22]:

$$
\begin{aligned}
& \frac{d g_{\kappa}(\epsilon, r)}{d r}=-\frac{\kappa}{r} g_{\kappa}(\epsilon, r)+\frac{\epsilon-V+m_{e} c^{2}}{c \hbar} f_{\kappa}(\epsilon, r) \\
& \frac{d f_{\kappa}(\epsilon, r)}{d r}=-\frac{\epsilon-V-m_{e} c^{2}}{c \hbar} g_{\kappa}(\epsilon, r)+\frac{\kappa}{r} f_{\kappa}(\epsilon, r)
\end{aligned}
$$

The expression of the NME can be written as a sum of three components [10]-[24]:

$$
M^{0 v}=M_{G T}^{0 v}-\left(\frac{g_{V}}{g_{A}}\right)^{2} \cdot M_{F}^{0 v}-M_{T}^{0 v}
$$

where $M_{G T}^{0 v}, M_{F}^{0 v}$ and $M_{T}^{0 v}$ are components associated with the matrix elements of the GamowTeller $(G T)$, Fermi $(F)$ and Tensor( $T)$ DBD operators, respectively. These are defined as follows:

$$
M_{\alpha}^{0 v}=\sum_{m, n}\left\langle 0_{f}^{+}\left\|\tau_{-m} \tau_{-n} O_{m n}^{\alpha}\right\| 0_{i}^{+}\right\rangle
$$

where $O_{m n}^{\alpha}$ are DBD transition operators $(\alpha=G T, F, T)$ and the summation is over all the nucleon states. The NME calculations can be divided in the computation of two quantities, the two-body transitions densities and the reduced matrix elements of the two-body transition operators $O^{\alpha}$. The most difficult is the computation of the radial part of the two-body transition operators, which contains 
the neutrino potentials. These potentials are defined by integrals of momentum carried by the virtual neutrino exchanged between the two nucleons inside the nucleus [10], [23]-[24]:

$$
\begin{gathered}
H_{\alpha}(r)=\frac{2 R}{\pi} \int_{0}^{\infty} j_{i}(q r) \frac{h_{\alpha}(q)}{\omega} \frac{1}{\omega+\langle E\rangle} q^{2} d q \\
\equiv \int_{0}^{\infty} j_{i}(q r) V_{\alpha}(q) q^{2} d q
\end{gathered}
$$

where $R=r_{0} A^{1 / 3} \mathrm{fm}\left(r_{0}=1.2 \mathrm{fm}\right), \omega=\sqrt{q^{2}+m_{v}^{2}}$ is the neutrino energy and $j_{n}(q r)$ is the spherical Bessel function ( $\mathrm{i}=0,0$ and 2 for GT, F, and T, respectively). Usually, in $0 v \beta \beta$ calculations one uses the closure approximation which consists in replacing the energies of the excited states of the intermediate odd-odd nucleus contributing to the decay, by an average value $\langle E\rangle$. This approximation works good in the case of $0 v \beta \beta$ decay modes (but not in the case of $2 v \beta \beta$ ) and simplifies much the calculations. The expressions of $h_{\alpha}(\alpha=G T, F, T)$ are [10]

$$
\begin{gathered}
h_{F}=G_{V}^{2}\left(q^{2}\right) \\
h_{G T}\left(q^{2}\right)=\frac{G_{A}^{2}\left(q^{2}\right)}{g_{A}^{2}}\left[1-\frac{2}{3} \frac{q^{2}}{q^{+} m_{\pi}^{2}}+\frac{1}{3}\left(\frac{q^{2}}{q^{2}+m_{\pi}^{2}}\right)^{2}\right]+ \\
+\frac{2}{3} \frac{G_{M}^{2}\left(q^{2}\right)}{g_{A}^{2}} \frac{q^{2}}{4 m_{p}^{2}}
\end{gathered}
$$

and

$$
\begin{gathered}
h_{T}\left(q^{2}\right)=\frac{G_{A}^{2}\left(q^{2}\right)}{g_{A}^{2}}\left[\frac{2}{3} \frac{q^{2}}{q^{2}+m_{\pi}^{2}}-\frac{1}{3}\left(\frac{q^{2}}{q^{2}+m_{\pi}^{2}}\right)^{2}\right]+ \\
+\frac{1}{3} \frac{G_{M}^{2}\left(q^{2}\right)}{g_{A}^{2}} \frac{q^{2}}{4 m_{p}^{2}}
\end{gathered}
$$

where $m_{\pi}$ is the pion mass, $m_{p}$ is the proton mass and

$$
G_{M}\left(q^{2}\right)=\left(\mu_{p}-\mu_{n}\right) G_{V}\left(q^{2}\right)
$$

with $\left(\mu_{p}-\mu_{n}\right)=4.71$.

The expressions (14)-(17) include nuclear ingredients that have to be taken into account for a precise computation of the NME, such as the inclusion of higher order currents in the nuclear interaction (HOC) and finite nucleon size effect (FNS). Inclusion of HOC effects brings additional terms in the $H_{G T}$ and $H_{F}$ components and apparition of the $H_{T}$ component in the expressions of the neutrino potentials. FNS effect is taken into account through $G_{V}$ and $G_{A}$ form factors [10], [12]:

$$
G_{A}\left(q^{2}\right)=g_{A}\left(\frac{\Lambda_{A}^{2}}{\Lambda_{A}^{2}+q^{2}}\right)^{2}, G_{V}\left(q^{2}\right)=g_{V}\left(\frac{\Lambda_{V}^{2}}{\Lambda_{V}^{2}+q^{2}}\right)^{2}
$$

For the vector and axial coupling constants many calculations take $g_{V}=1$ and the unquenched value $g_{A}=1.25$, while the values of the vector and axial vectors form factors are $\Lambda_{V}=850 \mathrm{MeV}$ and $\Lambda_{A}=1086 \mathrm{MeV}[1]$, respectively. 
To compute the radial matrix elements $\left\langle n l\left|H_{\alpha}\right| n^{\prime} l^{\prime}\right\rangle$ one uses harmonic oscillators w. f. $\psi_{n l}(l r)$ and $\psi_{n^{\prime} l^{\prime}}(r)$ corrected by a factor $[1+f(r)]$, in order to take into account the short range correlations (SRC) induced by the nuclear interaction:

$$
\psi_{n l}(r) \rightarrow[1+f(r)] \psi_{n l}(r)
$$

For the correlation function we take the functional form

$$
f(r)=-c \cdot e^{-a r^{2}}\left(1-b r^{2}\right)
$$

where $a, b$ and $c$ are constants that have particular values for different parameterizations [10], [25][26]. For $c=1$, we get the Jastrow prescription of inclusion of the SRC effects, and in this case the $a$ and $b$ constants are given by the Miller-Spencer (MS) parametrization [18].

\section{Numerical results and discussions}

The NME calculations are performed with a new ShM code [23]. In a previous paper [24] we shortly presented a new improved (fast, efficient) ShM code that reduces substantially the computing time of calculation of the two-body matrix elements (TBME) of the transition operators for the $0 v \beta \beta$ decay. It incorporates all the relevant nuclear effects. The radial NME are double integrals, over coordinates and momentum, that require long CPU time to be performed. The main improvement of the code consists in performing analytically the radial integrals over the coordinates, by rearranging the terms depending on " $r$ " in the TBME expression and using recurrence properties of the Laguerre polynomials and Bessel functions. Therefore, only the integration over the momentum remains to be performed numerically. Testing this code we found it about 30 times faster than our previous code [12], for the same calculations, and it can be of much help for investigating the quenching of the Gammow-Teller (GT) operator in ShM calculations of the NME, in large model spaces. The computation of the NME is performed with nuclear ingredients chosen according to a general consensus, as follows. The short range correlations between nucleons are introduced according to the CD-Bonn parametrization [10], the tensor contribution, due to the inclusion of HOC effects, is taken into account, the FNS effect is involved in calculation through the form factors $g_{V}$ and $g_{A}$ with the cut-off parameters $\Lambda_{V}=0.85 \mathrm{GeV}$ and $\Lambda_{A}=1.09 \mathrm{GeV}$. For $g_{A}$ we adopt the unquenched value 1.25 and the nuclear radius $R=r_{0} A^{1 / 3}$ is calculated with the constant $r_{0}=1.2 \mathrm{fm}$.

To compute PSF for DBD modes we obtained the w.f. of the electrons emitted in the decay, which are distorted by the Coulomb potential of the nucleus. We use exact Dirac electron w.f. with the inclusion of the FNS and screening effects. This last effect was taken into account by multiplying the expression of the Coulomb potential with a function $\phi(r)$, which is solution of the Thomas Fermitype equation [20], [22]. In addition to other previous calculations, we use a Coulomb-type potential derived from a realistic proton density distribution in nucleus. Also, we develop own routines for solving the Dirac equations and integrate the PSF expressions with improved numerical accuracy as compared with older codes, and we use new $Q$-values reported in ref. [27].

Our results are presented in In Tables I and II. In Table I upper limits for the electron-neutrino mass parameter $m_{v}$, associated with the light LH neutrino exchange mechanism, are derived in the case of three isotopes, ${ }^{48} \mathrm{Ca},{ }^{76} \mathrm{Ge}$ and ${ }^{82} \mathrm{Se}$, together with corresponding NME and $Q$-values and experimental lifetime limits. The effective NN interactions used are: GXPF1A [28] for ${ }^{48} \mathrm{Ca}$ and JUN45 [29] for ${ }^{76} \mathrm{Ge}$ and ${ }^{82} \mathrm{Se}$. As seen from the Table I, the most stringent upper limit for the neutrino mass parameter, which is related to the electron-neutrino absolute mass, is obtained from the study of DBD of ${ }^{76} \mathrm{Ge}$ and is of $0.27 \mathrm{eV}$. This is due both to the accurate calculation of the NME 
(with advanced ShM methods) and to the experimental lower limit of the half-life $\left(\sim 2 \times 10^{25} \mathrm{yr}\right)$, in the case of this isotope. Indeed, the DBD experiments on ${ }^{76} \mathrm{Ge}$, besides those on ${ }^{136} \mathrm{Xe}$ are at present the most sensitives ones and provide us with the largest lower limits for $0 v \beta \beta$ decay half-lives. These limits, of $\sim 0.2 \mathrm{eV}$, for the "light" neutrino parameters can be checked by the next generation of DBD experiments. In Table II upper limits of the neutrino parameter (coupling strength) $\eta_{N}$, associated with the exchange of a heavy RH neutrino mechanism are presented. Again, due to the same reason, the most stringent upper limits for the "heavy" neutrino mass parameter is extracted from the ${ }^{76} \mathrm{Ge}$ isotope.

Table 1. Upper limits of the Majorana light neutrino mass parameter together with the other components of the DBD lifetime: $Q$ values, experimental lifetime limits, PSF and NME.

\begin{tabular}{lccccc}
\hline & $Q[\mathrm{MeV}]$ & $T_{\text {exp }}^{0 v}[\mathrm{yr}]$ & $G^{0 v}\left[\mathrm{yr}^{-1}\right]$ & $M_{v}^{0 v}$ & $\left\langle m_{v}\right\rangle[\mathrm{eV}]$ \\
${ }^{48} \mathrm{Ca}$ & 4.272 & $>5.810^{22}[30]$ & $2.46 \mathrm{E}-14$ & 0.81 & $<9.62$ \\
${ }^{76} \mathrm{Ge}$ & 2.039 & $>2.110^{25}[31]$ & $2.37 \mathrm{E}-15$ & 3.13 & $<0.27$ \\
\hline${ }^{82} \mathrm{Se}$ & 2.995 & $>3.610^{23}[32]$ & $1.01 \mathrm{E}-14$ & 2.88 & $<1.19$ \\
\hline
\end{tabular}

Table 2. Upper limits of the Majorana heavy neutrino mass parameter together with experimental lifetime limits, PSF and NME.

\begin{tabular}{lcccc}
\hline & $T_{e x p}^{0 v}[y r]$ & $G^{0 v}\left[y r^{-1}\right]$ & $M_{N}^{0 v}$ & $\left\langle\eta_{N}\right\rangle[e V]$ \\
${ }^{48} \mathrm{Ca}$ & $>5.810^{22}[30]$ & $2.46 \mathrm{E}-14$ & 70.2 & $<3.77 \cdot 10^{-7}$ \\
${ }^{76} \mathrm{Ge}$ & $>2.110^{25}[31]$ & $2.37 \mathrm{E}-15$ & 199.2 & $<0.22 \cdot 10^{-7}$ \\
${ }^{82} \mathrm{Se}$ & $>3.610^{23}[32]$ & $1.01 \mathrm{E}-14$ & 184.5 & $<0.90 \cdot 10^{-7}$ \\
\hline
\end{tabular}

\section{Conclusions}

In this work I make first a short review on the challenges encountered in the computation of the PSF and NME, the two key ingredients in the study of the $0 v \beta \beta$ decay mode. Then, I present new constraints of the neutrino mass parameters associated with the "light" and "heavy" neutrino exchange mechanisms of occurrence of the $0 v \beta \beta$ decay mode. The NME and PSF for three isotopes, ${ }^{48} \mathrm{Ca}$, ${ }^{76} \mathrm{Ge}$ and ${ }^{82} \mathrm{Se}$, are calculated with advanced codes developed by our group, while the most recent experimental limits for $0 v \beta \beta$ lifetimes were taken from literature. The most strigent upper limits for the neutrino parameters were obtained for the ${ }^{76} \mathrm{Ge}$ isotope, for which both accurate calculations for NME and PSF, and the largest experimental lifetime value, exist.

\section{Acknowledgment}

This work was done with the support of the MENCS and UEFISCDI through the project IDEI-PCE3-0318, contract Nr. 58/28.10/2011.

\section{References}

[1] F. T. Avignon, S.R. Elliott and J. Engel, Rev. Mod. Phys. 80, 4812008.

[2] H. Ejiri. Prog. Part. Nucl. Phys., 4, 249 (2010). 
[3] J. Vergados, H. Ejiri and F. Simkovic, Rep. Prog. Phys. 75, 106301 (2012).

[4] W. Rodejohann, J.Phys. G: Nucl. Part. Phys. 39, 124008 (2012).

[5] A. Faessler, V. Rodin, F. Simkovic, J. Phys. G 39, 124006 (2012).

[6] P. Vogel, J.Phys. G: Nucl. Part. Phys. 39, 124002 (2012).

[7] S. Stoica and H.V. Klapdor-Kleingrothaus, Nucl. Phys.A 694 (2001) 269.

[8] M. Kortelainen and J. Suhonen, Phys. Rev. C 75, 051303 (2007); Phys. Rev. C 76, 024315 (2007).

[9] F. Simkovic, A. Faessler, V.A. Rodin, P. Vogel and J. Engel, Phys. Rev. C 77, 045503 (2008)

[10] F. Simkovic, A. Faessler, H. Muther, V. Rodin, and M. Stauf, Phys. Rev. C 79, 055501 (2009).

[11] J. Menendez, A. Poves, E. Caurier, F. Nowacki, and A. Poves, Nuclear Physics A 818139 (2009).

[12] M. Horoi and S. Stoica, Phys. Rev. 81, 024321 (2010).

[13] J. Barea and F. Iachello, Phys. Rev. C 79, 044301 (2009).

[14] J. Barea, J. Kotila, F. Iachello, Phys. Rev. C 87, 014315 (2013).

[15] P.K. Rath, R. Chandra, K. Chaturvedi, P.K. Raina, J.G. Hirsch, Phys. Rev. C 82, 064310 (2010).

[16] T.R. Rodriguez and G. Martinez-Pinedo, Phys. Rev. Lett 105, 252503 (2010).

[17] M. Doi, T. Kotani and E. Takasugi, Prog. Theor. Phys. Suppl. 83, 1 (1985).

[18] T. Tomoda, Rep. Prog. Phys. 54, 53 (1991).

[19] J. Suhonen and O. Civitarese, Phys. Rep. 300, 123 (1998).

[20] J. Kotila and F. Iachello, Phys. Rev. C 85, 034316 (2012).

[21] S. Stoica and M. Mirea, Phys. Rev. C 88, 037303 (2013).

[22] M. Mirea, T. E. Pahomi and S. Stoica, Rom. Rep. Phys. 67, 872 (2015).

[23] A. Neacsu, S. Stoica, J. Phys. G:Nucl. Part. Phys. 41, 015201 (2014).

[24] A. Neacsu, S. Stoica and M. Horoi, Phys. Rev. C 86, 067304 (2012).

[25] C. Giusti, H. Muther, F. D. Pacati, and M. Stauf, Phys. Rev. C 60, 054608 (1999).

[26] H. Muther and A Polls, Phys. Rev. C 61, 014304 (1999) Part. Nucl. Phys. 45, 243 (2000).

[27] M. Wang, G. Audi, A.H. Wapstra, F.G. Kondev, M. MacCornick, X. Xu, B. Pfeiffer, Chin. Phys. C 36, 1603 (2012).

[28] M. Honma, T. Otsuka, B. A. Brown, and T. Mizusaki, Eur. Phys. J. A 25, Suppl. 1, 499 (2005).

[29] M. Honma, T. Otsuka, T. Mizusaki and M. Hjorth-Jensen, Phys. Rev. C 80, 064323 (2009).

[30] S. Umehara et al., Phys Rev C 78, 058501 (2008).

[31] C. Macolino et al. (GERDA Collaboration), Mod. Phys. Lett. A 29, 1430001 (2014).

[32] A. S. Barabash, and Brudanin V. B., Phys. At. Nucl. 74, 312 (2011). 